\title{
New Constructions of Resilient Boolean Functions with Maximal Nonlinearity
}

\author{
Yuriy Tarannikov \\ Mech. \& Math. Department \\ Moscow State University \\ 119899 Moscow, Russia \\ yutaran@mech.math.msu.su, taran@vertex.inria.msu.ru
}

\begin{abstract}
In this paper we develop a technique that allows to obtain new effective constructions of highly resilient Boolean functions with high nonlinearity. In particular, we prove that the upper bound $2^{n-1}-2^{m+1}$ on nonlinearity of $m$-resilient $n$-variable Boolean functions is achieved for $0.6 n-1 \leq m \leq n-2$.
\end{abstract}

Keywords. Stream cipher, Boolean function, nonlinear combining function, correlation-immunity, resiliency, nonlinearity.

\section{Introduction}

One of the most general types of stream cipher systems is several Linear Feedback Shift Registers (LFSRs) combined by nonlinear Boolean function. This function must satisfy certain criteria to resist different attacks (in particular, correlation attacks suggested by Siegenthaler [18] and different types of linear attacks). The following factors are considered as important properties of Boolean functions for using in stream cipher applications.

1. Balancedness. A Boolean function must output zeroes and ones with the same probabilities.

2. Good correlation-immunity (of order $m$ ). The output of Boolean function must be statistically independent of combination of any $m$ its inputs. A balanced correlation-immune of order $m$ Boolean function is called $m$-resilient.

3. Good nonlinearity. The Boolean function must be at the sufficiently high distance from any affine function.

Other important factors are large algebraic degree and simple implementation in hardware.

The variety of criteria and complicated trade-offs between them caused the next approach: to fix one or two parameters and try to optimize others. The most general model is when researchers fix the parameters $n$ (number of variables) and $m$ (order of correlation-immunity) and try to optimize some other cryptographically important parameters. Here we can call the works [16], [2], [7], [4] [8], [9], [10], [12], [19], [20], [22].

The present paper continues the investigations in this direction and gives new results. In Section 2 we give preliminary concepts and notions. In Section 3 we 
give a brief review of the investigations on the problem of maximal nonlinearity for $n$-variable $m$-resilient Boolean function. In Section 4 we discuss a concept of a linear and a pair of quasilinear variables which works in the following sections. In Section 5 we present our main construction method. This method is a generalization of a method described in [19] and [20]. This method allows to construct recursively the functions with good cryptographic properties using the functions with good cryptographic properties and smaller number of variables. The method is based on the existence of a proper matrix with prescribed properties. In Section 6 we give some examples of proper matrices and obtain new results on the maximal nonlinearity $\operatorname{nlmax}(n, l)$ of $m$-resilient functions on $V^{n}$. Namely, we prove that $\operatorname{nlmax}(n, m)=2^{n-1}-2^{m+1}$ for $\frac{5 n-14}{8} \leq m \leq n-2$ and for $0.6 n-1 \leq m \leq n-2$. In Section 7 we give some remarks on the combinatorial problem connected with proper matrices and give a geometrical interpretations of proper matrices.

\section{Preliminary Concepts and Notions}

We consider $V^{n}$, the vector space of $n$ tuples of elements from $G F(2)$. A Boolean function is a function from $V^{n}$ to $G F(2)$. The weight wt $(f)$ of a function $f$ on $V^{n}$ is the number of vectors $x$ on $V^{n}$ such that $f(x)=1$. A function $f$ is said to be balanced if $w t(f)=w t(f \oplus 1)$. Obviously, if a function $f$ on $V^{n}$ is balanced then $w t(f)=2^{n-1}$. A subfunction of the Boolean function $f$ is a function $f^{\prime}$ obtained by substitution some constants for some variables in $f$. If we substitute in the function $f$ the constants $\sigma_{i_{1}}, \ldots, \sigma_{i_{s}}$ for the variables $x_{i_{1}}, \ldots, x_{i_{s}}$ respectively then the obtained subfunction is denoted by $f_{x_{i_{1}}, \ldots, x_{i_{s}}}^{\sigma_{i_{1}}, \ldots, \sigma_{i_{s}}}$. If a variable $x_{i}$ is not substituted by constant then $x_{i}$ is called a free variable for $f^{\prime}$.

It is well known that a function $f$ on $V^{n}$ can be uniquely represented by a polynomial on $G F(2)$ whose degree is at most $n$. Namely,

$$
f\left(x_{1}, \ldots, x_{n}\right)=\bigoplus_{\left(a_{1}, \ldots, a_{n}\right) \in V^{n}} g\left(a_{1}, \ldots, a_{n}\right) x_{1}^{a_{1}} \ldots x_{n}^{a_{n}}
$$

where $g$ is also a function on $V^{n}$. This polynomial representation of $f$ is called the algebraic normal form (briefly, ANF) of the function and each $x_{1}^{a_{1}} \ldots x_{n}^{a_{n}}$ is called a term in ANF of $f$. The algebraic degree of $f$, denoted by $\operatorname{deg}(f)$, is defined as the number of variables in the longest term of $f$. The algebraic degree of variable $x_{i}$ in $f$, denoted by $\operatorname{deg}\left(f, x_{i}\right)$, is the number of variables in the longest term of $f$ that contains $x_{i}$. If $\operatorname{deg}\left(f, x_{i}\right)=1$, we say that $x_{i}$ is a linear variable in $f$. The term of length 1 is called a linear term. If $\operatorname{deg}(f) \leq 1$ then $f$ is called an affine function.

The Hamming distance $d\left(x^{\prime}, x^{\prime \prime}\right)$ between two vectors $x^{\prime}$ and $x^{\prime \prime}$ is the number of components where vectors $x^{\prime}$ and $x^{\prime \prime}$ differ. For two Boolean functions $f_{1}$ and $f_{2}$ on $V^{n}$, we define the distance between $f_{1}$ and $f_{2}$ by $d\left(f_{1}, f_{2}\right)=\#\{x \in$ $\left.V^{n} \mid f_{1}(x) \neq f_{2}(x)\right\}$. The minimum distance between $f$ and the set of all affine functions is called the nonlinearity of $f$ and denoted by $n l(f)$. 
A Boolean function $f$ on $V^{n}$ is said to be correlation-immune of order $m$, with $1 \leq m \leq n$, if the output of $f$ and any $m$ input variables are statistically independent. This concept was introduced by Siegenthaler [17]. In equivalent nonprobabilistic formulation the Boolean function $f$ is called correlation-immune of order $m$ if $w t\left(f^{\prime}\right)=w t(f) / 2^{m}$ for any its subfunction $f^{\prime}$ of $n-m$ variables. A balanced $m$ th order correlation immune function is called an $m$-resilient function. In other words the Boolean function $f$ is called $m$-resilient if $w t\left(f^{\prime}\right)=2^{n-m-1}$ for any its subfunction $f^{\prime}$ of $n-m$ variables. From this point of view we can consider formally any balanced Boolean function as 0-resilient (this convention is accepted in [1], [9], [12]) and an arbitrary Boolean function as (-1)-resilient. The concept of an $m$-resilient function was introduced in [3].

Siegenthaler's Inequality [17] states that if the function $f$ is a correlation-immune function of order $m$ then $\operatorname{deg}(f) \leq n-m$. Moreover, if $f$ is an $m$-resilient, $m \leq n-2$, then $\operatorname{deg}(f) \leq n-m-1$.

The next lemma is well-known.

Lemma 1. Let $f\left(x_{1}, \ldots, x_{n}\right)$ be a Boolean function represented in the form

$f\left(x_{1}, \ldots, x_{n}\right)=\bigoplus_{\left(\sigma_{1}, \ldots, \sigma_{l}\right)}\left(x_{1} \oplus \sigma_{1}\right) \ldots\left(x_{l} \oplus \sigma_{l}\right) f\left(\sigma_{1} \oplus 1, \ldots, \sigma_{l} \oplus 1, x_{l+1}, \ldots, x_{n}\right)$.

Suppose that all $2^{l}$ subfunctions $f\left(\sigma_{1} \oplus 1, \ldots, \sigma_{l} \oplus 1, x_{l+1}, \ldots, x_{n}\right)$ are $m$-resilient. Then the function $f$ is an m-resilient too.

The Lemma 1 was proved in a lot of papers including (for $l=1$ ) the pioneering paper of Siegenthaler (Theorem 2 in [17]). General case follows immediately from the case $l=1$.

\section{The Problem of Maximal Nonlinearity for Resilient Functions}

Let $m$ and $n$ be integers, $-1 \leq m \leq n$. Denote by $n \operatorname{lmax}(n, m)$ the maximal possible nonlinearity of $m$-resilient Boolean function on $V^{n}$. It is well-known that the nonlinearity of a Boolean function does not exceed $2^{n-1}-2^{\frac{n}{2}-1}$ [15]. Thus, $\operatorname{nlmax}(n,-1) \leq 2^{n-1}-2^{\frac{n}{2}-1}$. This value can be achieved only for even $n$. The functions with such nonlinearity are called bent functions. Thus, for even $n$ we have $n \operatorname{lmax}(n,-1)=2^{n-1}-2^{\frac{n}{2}-1}$. It is known $[13,14,7]$ that for odd $n$, $n \leq 7, \operatorname{nlmax}(n,-1)=2^{n-1}-2^{(n-1) / 2}$, and for odd $n, n \geq 15$, the inequality $n \max (n,-1)>2^{n-1}-2^{(n-1) / 2}$ holds. Bent functions are nonbalanced always, so, for balanced (0-resilient) $n$-variable function $f$ we have $n l(f)<2^{n-1}-$ $2^{\frac{n}{2}-1}$, and $n \operatorname{lmax}(n, m)<2^{n-1}-2^{\frac{n}{2}-1}$ for $m \geq 0$. If $f$ is $n$-variable $m$-resilient function, $m \geq n-2$, then by Siegenthaler's Inequality [17] $\operatorname{deg}(f) \leq 1$, so $n \max (n, m)=0$. For some small values of parameters $n$ and $m$ exact values of maximal nonlinearity are known. The latest collection of such values is given in [10]. The upper bound $\operatorname{nlmax}(n, m) \leq 2^{n-1}-2^{m+1}$ for $m \leq n-1$ was proven independently in [10], [19] and [23], all three these manuscripts were submitted 
to Crypto 2000 although only the first was accepted). In [19] and [20] an effective construction of $m$-resilient function on $V^{n}$ with nonlinearity $2^{n-1}-2^{m+1}$ was given. This construction gives that $\operatorname{nlmax}(n, m)=2^{n-1}-2^{m+1}$ for $\frac{2 n-7}{3} \leq m \leq$ $n-2$. The construction of [19] and [20] was slightly modified in [11]. The last modification follows that $\operatorname{nlmax}(n, m)=2^{n-1}-2^{m+1}$ for $\frac{2 n-8}{3} \leq m \leq n-2$. In [10] it was proved that the nonlinearity of $m$-resilient function on $V^{n}$ is divided by $2^{m+1}$. Also in [10] it was proved that $\operatorname{nlmax}(n, m) \leq 2^{n-1}-2^{n / 2-1}-2^{m+1}$ for $m<(n / 2)-2$ (for the case of odd $n$ a bit more strong bound was given).

\section{On Linear and Quasilinear Variables}

In this section we recall the concepts of linear and quasilinear variables. The last concept was introduced in [19].

Recall that a variable $x_{i}$ is called a linear for a function $f=f\left(x_{1}, \ldots, x_{i-1}\right.$, $\left.x_{i}, x_{i+1}, \ldots, x_{n}\right)$ if $\operatorname{deg}\left(f, x_{i}\right)=1$. Also we say that a function $f$ depends on a variable $x_{i}$ linearly. If a variable $x_{i}$ is linear for a function $f$ we can represent $f$ in the form

$$
f\left(x_{1}, \ldots, x_{i-1}, x_{i}, x_{i+1}, \ldots, x_{n}\right)=g\left(x_{1}, \ldots, x_{i-1}, x_{i+1}, \ldots, x_{n}\right) \oplus x_{i} .
$$

Other equivalent definition of a linear variable is that a variable $x_{i}$ is linear for a function $f$ if $f\left(x^{\prime}\right) \neq f\left(x^{\prime \prime}\right)$ for any two vectors $x^{\prime}$ and $x^{\prime \prime}$ that differ only in $i$ th component. By analogy with the last definition we give a new definition for a pair of quasilinear variables.

Definition 1. We say that a Boolean function $f=f\left(x_{1}, \ldots, x_{n}\right)$ depends on a pair of its variables $\left(x_{i}, x_{j}\right)$ quasilinearly if $f\left(x^{\prime}\right) \neq f\left(x^{\prime \prime}\right)$ for any two vectors $x^{\prime}$ and $x^{\prime \prime}$ of length $n$ that differ only in ith and $j$ th components. A pair $\left(x_{i}, x_{j}\right)$ in this case is called a pair of quasilinear variables in $f$.

The proof of the next lemma is given in [19] and [20].

Lemma 2. Let $f\left(x_{1}, \ldots, x_{n}\right)$ be a Boolean function. Then $\left(x_{i}, x_{j}\right), i<j$, is a pair of quasilinear variables in $f$ iff $f$ can be represented in the form

$$
f\left(x_{1}, \ldots, x_{n}\right)=g\left(x_{1}, \ldots, x_{i-1}, x_{i+1}, \ldots, x_{j-1}, x_{j+1}, \ldots, x_{n}, x_{i} \oplus x_{j}\right) \oplus x_{i} .
$$

The next lemmas are obvious.

Lemma 3. Let $f\left(x_{1}, \ldots, x_{n}\right)$ be a Boolean function. If $f$ depends on some variable $x_{i}$ linearly then $f$ is balanced.

Lemma 4. $f\left(x_{1}, \ldots, x_{n}\right)$ be a Boolean function. If $f$ depends on some variables $x_{i_{1}}, x_{i_{2}}, \ldots, x_{i_{s}}$ linearly then $f$ is $(s-1)$-resilient.

Note that Lemma 4 agrees with our assumption that a balanced function is 0 -resilient, and an arbitrary Boolean function is (-1)-resilient. (In the last case $s=0$.) 
Lemma 5. Let $f\left(x_{1}, \ldots, x_{n}\right)$ be a Boolean function. If $f$ depends on some pair of variables $\left(x_{i}, x_{j}\right)$ quasilinearly then $f$ is balanced.

Lemma 6. Let $f\left(x_{1}, \ldots, x_{n}, x_{n+1}\right)=f\left(x_{1}, \ldots, x_{n}\right) \oplus c x_{n+1}$ where $c \in\{0,1\}$. Then $n l(f)=2 n l(g)$.

Lemma 7. Let $f\left(x_{1}, \ldots, x_{n}\right)$ be a Boolean function on $V^{n}$ and $f$ depends on some pair of variables $\left(x_{i}, x_{j}\right)$ quasilinearly. Then $n l(f)=2 n l(g)$ where $g$ is a function used in the representation of $f$ in Lemma 2.

Lemma 8. Let $f_{1}$ and $f_{2}$ be two Boolean functions on $V^{n}$. Moreover, there exist variables $x_{i}$ and $x_{j}$ such that $f_{1}$ depends on a pair of variables $\left(x_{i}, x_{j}\right)$ quasilinearly whereas $f_{2}$ depends on the variables $x_{i}$ and $x_{j}$ linearly. Let $l$ be an arbitrary affine function on $V^{n}$. Then at least one of two functions $f_{1} \oplus l$ and $f_{2} \oplus l$ is balanced.

Proof. Let $l=\bigoplus_{r=1}^{n} u_{r} x_{r} \oplus u_{0}, u_{r} \in\{0,1\}, r=0,1, \ldots, n$. If $u_{i}=0$ (correspondently, $\left.u_{j}=0\right)$ then $f_{2} \oplus l$ depends on the variable $x_{i}\left(x_{j}\right)$ linearly, therefore the function $f_{2} \oplus l$ is balanced. The remained case is $u_{i}=u_{j}=1$. But here it is easy to see that the function $f_{1} \oplus l$ depends on a pair of variables $\left(x_{i}, x_{j}\right)$ quasilinearly, therefore $f_{1} \oplus l$ is balanced.

\section{$5 \quad$ A Method of Constructing}

Suppose that $f_{0}, f_{1}, \ldots, f_{2^{k}-1}$ are Boolean functions on $V^{n}$. We denote $f_{r}$ also as $f_{\sigma_{1} \ldots \sigma_{k}}$ where $\sigma_{1} \ldots \sigma_{k}$ is a binary representation of the number $r$. Suppose that $c=\left(c_{1}, \ldots, c_{k}\right)$ is an arbitrary binary vector. Put $s=\sum_{i=1}^{k} c_{i}$. We denote $X=\left\{x_{i} \mid i=1, \ldots, n\right\}, Y=\left\{y_{i} \mid i=1, \ldots, k\right\}, Z=\left\{z_{i} \mid c_{i}=1, i=1, \ldots, k\right\}$ (The set $Z$ contains the variables $z_{i}$ only for $i$ such that $c_{i}=1$ ). We define

$$
f(X, Y, Z)=\left(\bigoplus_{\left(\sigma_{1}, \ldots, \sigma_{k}\right) \in V^{k}}\left(\prod_{i=1}^{k}\left(y_{i} \oplus c_{i} z_{i} \oplus \sigma_{i}\right)\right) f_{\sigma_{1} \ldots \sigma_{k}}(X)\right) \oplus \bigoplus_{i=1}^{k} c_{i} z_{i}
$$

By construction the function $f$ in $(2)$ depends on $n+k+s$ variables. Below we formulate some properties of construction (2).

Remark. Some details of construction (2) can be understood more easily if we put $c_{1}=\ldots=c_{s}=1, c_{s+1}=\ldots=c_{k}=0$. But for an effective implementation it is important in some cases to vary the vector $c$.

Lemma 9. Suppose that all $2^{k}$ Boolean functions $f_{0}, f_{1}, \ldots, f_{2^{k}-1}$ in (2) are $m$-resilient. Then the function $f(X, Y, Z)$ is $(m+s)$-resilient.

Proof. Substitute in (2) arbitrary $m+s$ constants for arbitrary $m+s$ variables. We obtain some $(n+k-m)$-variable subfunction $f^{\prime}$. If $c_{i}=1$ for some $i$ and 
if both variables $y_{i}$ and $z_{i}$ are free in $f^{\prime}$ then the pair of variables $\left(y_{i}, z_{i}\right)$ is a quasilinear pair in $f^{\prime}$ therefore by Lemma 5 the subfunction $f^{\prime}$ is balanced). Thus, we can assume that for each $i$ such that $c_{i}=1$ at least one of two variables $y_{i}$ and $z_{i}$ is substituted by constant. Then at most $m$ variables from $X$ are substituted by constants in (2). All functions $f_{0}, f_{1}, \ldots, f_{2^{k}-1}$ are balanced, therefore the function $f(X, Y, Z)$ is balanced too. We have proved that an arbitrary $(n+k-m)$ variable subfunction of $f(X, Y, Z)$ is balanced.

Lemma 10. Suppose that the nonlinearity of all $2^{k}$ Boolean functions $f_{0}, f_{1}$, $\ldots, f_{2^{k}-1}$ in (2) is at least $N_{0}$. Moreover, for any two functions $f_{r_{1}}$ and $f_{r_{2}}$, $0 \leq r_{1} \neq r_{2} \leq 2^{k}-1$, there exists a pair of variables $\left(x_{i}, x_{j}\right)$ such that one of these two functions, say $f_{r_{1}}$ depends linearly on the variables $x_{i}$ and $x_{j}$ whereas another function $f_{r_{2}}$ depends quasilinearly on the pair $\left(x_{i}, x_{j}\right)$. Then $n l(f) \geq$ $2^{s}\left(2^{n-1}\left(2^{k}-1\right)+N_{0}\right)$.

Proof. It is obvious that if we replace $c_{i}=0$ by $c_{i}=1$ then we multiplate the nonlinearity by 2 (adding new variable). Thus we can assume that $s=0$. Consider an arbitrary affine function $l$. Denote $l_{r}=l_{y_{1}, \ldots 1, \ldots, \sigma_{k} \oplus 1}^{\sigma_{1} \oplus 1}$ where $\sigma_{1} \ldots \sigma_{k}$ is a binary representation of the number $r$. Note that for any $r=0, \ldots, 2^{k}-1$, we have $l_{r}=l_{0}$ or $l_{r}=l_{0} \oplus 1$. Then $d(f, l)=\sum_{r=0}^{2^{k}-1} d\left(f_{r}, l_{r}\right)$. By Lemma 8 and the hypothesis of this lemma we have that $d\left(f_{r}, l_{r}\right) \neq 2^{n-1}$ for at most one value of $r$. Thus $d(f, l) \geq 2^{n-1}\left(2^{k}-1\right)+N_{0}$. An affine function $l$ was chosen arbitrary. Therefore $n l(f) \geq 2^{n-1}\left(2^{k}-1\right)+N_{0}$.

The construction (2) is a generalization of the construction in [19] and [20] where only the case $k=1$ is considered.

The problem is to find the functions $f_{0}, f_{1}, \ldots, f_{2^{k}-1}$ with desirable distribution of linear and quasilinear variables. Below we give some approach that allows to construct such systems of functions.

Definition 2. Let $B=\left(b_{i j}\right)$ be $\left(2^{k} \times p\right)$ matrix of $2^{k}$ rows and $p$ columns with entries from the set $\{1,2, *\}$. Let $k_{0}$ and $t$ be positive integers. We assume that

(i) for every two rows $i_{1}$ and $i_{2}$ there exists a column $j$ such that $b_{i_{1} j}=1$, $b_{i_{2} j}=2$ or $b_{i_{1} j}=2, b_{i_{2} j}=1$.

(ii) for every row $i$ the inequality $\sum_{j=1}^{p} b_{i j} \leq t$ holds (a sign $*$ does not give an influence to these sums).

(iii) in every row the number of ones does not exceed $k_{0}$.

If the matrix $B$ satisfies all properties (i), (ii), (iii) we say that $B$ is a proper $\left(k_{0}, k, p, t\right)$-matrix.

Definition 3. Let $F$ be a set of Boolean functions such that for every $s, 0 \leq$ $s \leq k$, the set $F$ contains an $(m+s)$-resilient function on $V^{n+s}$ with nonlinearity at least $2^{s}\left(2^{n-1}-2^{m+\lambda}\right)$ ( $\lambda$ is not necessary integer). Moreover, we assume that each $f_{s}$ contains $s$ disjoint pairs of quasilinear variables. Then we say that $F$ is a $S_{n, m, k, \lambda}$-system of Boolean functions. 
Remark. To provide an existence of a $S_{n, m, k, \lambda}$-system of Boolean functions it is sufficient to have only one $(m+k)$-resilient function $f$ on $V^{n+k}$ with nonlinearity at least $2^{k}\left(2^{n-1}-2^{m+\lambda}\right)$ that contains $k$ disjoint pairs of quasilinear variables. All other necessary functions of $S_{n, m, k, \lambda}$-system can be obtained from $f$ by substitutions of constants for the variables from different disjoint pairs of quasilinear variables. But note that the last way is not effective from the implementation point of view.

Lemma 11. There exists an $S_{2,-1,2,1}$-system of Boolean functions.

Proof. Put $f_{0}^{\prime}=x_{1} x_{2}, f_{1}^{\prime}=\left(x_{1} \oplus x_{2}\right) x_{3} \oplus x_{1}, f_{2}^{\prime}=\left(x_{1} \oplus x_{2}\right)\left(x_{3} \oplus x_{4}\right) \oplus x_{1} \oplus x_{3}$. It is easy to verify that $f_{s}^{\prime}, s=0,1,2$, is a $(-1+s)$-resilient function on $V^{2+s}$ with nonlinearity $2^{s}\left(2^{2-1}-2^{-1+1}\right)$, moreover, $f_{s}^{\prime}$ contains $s$ disjoint pairs of quasilinear variables.

Theorem 1. Suppose that there exists an $S_{n, m, k_{0}, \lambda}$-system of Boolean functions $F$ and there exists a proper $\left(k_{0}, k, p, t\right)$-matrix $B, n \geq 2 p-t$. Then there exists an $S_{n+k+t, m+t, k, \lambda}$-system of Boolean functions.

Proof. Consider the $i$ th row of the matrix $B, i=0,1, \ldots, 2^{k}-1$. Suppose that this row contains $s=s(i)$ ones. The matrix $B$ is a proper, therefore $s \leq k_{0}, s \leq t$. By assumption there exists an $(m+s)$-resilient function $f_{i}^{\prime}$ on $V^{n+s}$ that contains $s$ disjoint pairs of quasilinear variables with nonlinearity at least $2^{s}\left(2^{n-1}-2^{m+\lambda}\right)$. Add $t-s$ new linear variables to the function $f_{i}^{\prime}$. As a result we obtain the function $f_{i}^{\prime \prime}$ on $V^{n+t}$. It is easy to see that the function $f_{i}^{\prime \prime}$ is an $(m+t)$-resilient function with nonlinearity at least $2^{t}\left(2^{n-1}-2^{m+\lambda}\right)$, moreover $f_{i}^{\prime \prime}$ contains $s$ disjoint pairs of quasilinear variables and besides $t-s$ linear variables. Note that by the property (ii) of a proper matrix the value $t-s$ is not less than the number of 2's in $i$ th row of $B$ multiplied by 2 . By this way we construct the functions $f_{i}^{\prime \prime}$ on $V^{n+t}$ for every $i, i=0,1, \ldots, 2^{k}-1$. By assumption $n+t \geq 2 p$. Next, for every $i, i=0,1, \ldots, 2^{k}-1$, we permute the variables in $f_{i}^{\prime \prime}\left(x_{1}, \ldots, x_{n+t}\right)$ obtaining the function $f_{i}$ such that the function $f_{i}$ depends on a pair of variables $\left(x_{2 j-1}, x_{2 j}\right)$ quasilinearly if $b_{i j}=1$, and the function $f_{i}$ depends on the variables $x_{2 j-1}$ and $x_{2 j}$ linearly if $b_{i j}=2$. By the arguments given above we have sufficient numbers of quasilinear and linear variables for this procedure. Now we are ready to apply the construction (2). By means of this construction varying the number of ones in the vector $\left(c_{1}, \ldots, c_{k}\right)$ we obtain the functions $f\left(X, Y, Z_{s}\right), s=0,1, \ldots, k$. The function $f\left(X, Y, Z_{s}\right)$ by Lemmas 9 and 10 is an $(m+t+s)$-resilient function on $V^{n+k+t+s}$ with the nonlinearity at least $2^{s}\left(2^{n+k+t-1}-2^{m+t+\lambda}\right)$. Moreover, the function $f\left(X, Y, Z_{s}\right)$ contains $s$ disjoint pairs of quasilinear variables. Thus, we have constructed an $S_{n+k+t, m+t, k, \lambda}$-system of Boolean functions.

An application of the construction given in Theorem 1 we denote by

$$
S_{n, m, k_{0}, \lambda} T_{k_{0}, k, p, t}=S_{n+k+t, m+t, k, \lambda} .
$$

If we add new linear variable to an $m$-resilient function $f$ on $V^{n}$ then we obtain $(m+1)$-resilient function $f$ on $V^{n+1}$ with nonlinearity $2 n l(f)$. We denote this procedure by

$$
S_{n, m, 0, \lambda} T_{0,0,0,1}=S_{n+1, m+1,0, \lambda} .
$$




\section{Examples of Proper Matrices Effective for Our Construction and New Resilient Boolean Functions with Maximal Nonlinearity}

At first, we give some examples of proper matrices effective for the construction of Boolean functions with good combination of parameters. We denote a proper $\left(k_{0}, k, p, t\right)$-matrix by $B_{k_{0}, k, p, t}$.

$$
B_{1,1,1,2}=\left(\begin{array}{c}
2 \\
1
\end{array}\right), B_{2,2,2,4}=\left(\begin{array}{ccc}
2 & 2 \\
2 & 1 \\
1 & 2 \\
1 & 1
\end{array}\right), B_{3,2,3,3}=\left(\begin{array}{cccc}
2 & 1 & * \\
* & 2 & 1 \\
1 & * & 2 \\
1 & 1 & 1
\end{array}\right),
$$

It is easy to verify that all matrices given above are proper matrices with correspondent parameters.

The simplest example of a proper matrix is the matrix $B_{1,1,1,2}$. If $\frac{2 n-7}{3} \leq m \leq$ $n-3$ then the numbers $n$ and $m$ can be represented in the form $n=3 r+s+2$, $m=2 r+s-1$, where $r$ and $s$ are nonnegative integers (an existence of this representation as well as an existence of the representations in Theorems 2 and 3 can be proved by the arguments from the elementary arithmetic). By Lemma 11 there exists a system $S_{2,-1,2,1}$. We apply

$$
S_{2,-1,2,1}\left(T_{1,1,1,2}\right)^{r}\left(T_{0,0,0,1}\right)^{s}=S_{n, m, 0,1} .
$$


Therefore $n \operatorname{lmax}(n, m) \geq 2^{n-1}-2^{m+1}$ for $m \geq \frac{2 n-7}{3}$. In Section 3 it was pointed out that $n \operatorname{lmax}(n, m) \leq 2^{n-1}-2^{m+1}$ for $m \leq n-2$. Therefore $\operatorname{nlmax}(n, m)=$ $2^{n-1}-2^{m+1}$ for $\frac{2 n-7}{3} \leq m \leq n-2$. The above construction was given in [19] and $[20]$.

Theorem 2. $n \operatorname{lmax}(n, m)=2^{n-1}-2^{m+1}$ for $\frac{5 n-14}{8} \leq m \leq n-2$.

Proof. Let $n, m$ be integers. Note that $\left\lceil\frac{5 n-14}{8}\right\rceil \geq\left\lceil\frac{2 n-7}{3}\right\rceil$ for $n<17$. If $n \geq 17, m>n-8$, then $m \geq \frac{2 n-7}{3}$. If $n \geq 17, \frac{5 n-13}{8} \leq m \leq n-8$, then the numbers $n$ and $m$ can be represented in the form $n=8 r_{1}+3 r_{2}+s+17$, $m=5 r_{1}+2 r_{2}+s+9$, where $r_{1}, r_{2}$ and $s$ are nonnegative integers. We apply

$$
S_{2,-1,2,1} T_{2,2,2,4} T_{2,3,5,6}\left(T_{3,3,4,5}\right)^{r_{1}}\left(T_{1,1,1,2}\right)^{r_{2}}\left(T_{0,0,0,1}\right)^{s}=S_{n, m, 0,1} .
$$

If $n \geq 17, \frac{5 n-14}{8}=m$, then the numbers $n$ and $m$ can be represented in the form $n=8 r+22, m=5 r+12$, where $r$ is nonnegative integer. In this case we apply

$$
S_{2,-1,2,1} T_{2,2,2,4} T_{2,3,5,6}\left(T_{3,3,4,5}\right)^{r} T_{3,2,3,3}=S_{n, m, 2,1}
$$

Theorem 3. $n \operatorname{lmax}(n, m)=2^{n-1}-2^{m+1}$ for $0.6 n-1 \leq m \leq n-2$.

Proof. Let $n, m$ be integers. Note that $0.6 n-1 \geq \frac{2 n-7}{3}$ for $n \leq 20$. If $n \geq 20$, $m>n-9$, then $m \geq \frac{2 n-7}{3}$. If $n \geq 20,0.6 n-1 \leq m \leq n-9$, excepting the case $m=0.6 n-1, n \equiv 5 \quad(\bmod 10)$, then the numbers $n$ and $m$ can be represented in the form $n=10 r_{1}+8 r_{2}+3 r_{3}+s+20, m=6 r_{1}+5 r_{2}+2 r_{3}+s+11$, where $r_{1}, r_{2}, r_{3}$ and $s$ are nonnegative integers. We apply

$$
S_{2,-1,2,1} T_{2,2,2,4} T_{2,4,7,8}\left(T_{4,4,6,6}\right)^{r_{1}}\left(T_{3,3,4,5}\right)^{r_{2}}\left(T_{1,1,1,2}\right)^{r_{3}}\left(T_{0,0,0,1}\right)^{s}=S_{n, m, 0,1} .
$$

In the case $n=10 r+25, m=6 r+14$, where $r$ is a nonnegative integer we apply

$$
S_{2,-1,2,1} T_{2,2,2,4} T_{2,4,7,8}\left(T_{4,4,6,6}\right)^{r} T_{3,2,3,3}=S_{n, m, 2,1}
$$

Note that the best previous result [11] was that $\operatorname{nlmax}(n, m)=2^{n-1}-2^{m+1}$ for $\frac{2 n-8}{3} \leq m \leq n-2$. Since $\frac{5 n-14}{8}<\frac{2 n-8}{3}$ for $n>22$ it is easy to check that our constructions provide the best known result beginning with $n=25$.

\section{Some Remarks on Combinatorial Problem and Geometrical Interpretations}

If there exists a proper $(k, k, p, t)$-matrix then using the technique described in the previous section we can prove that $\operatorname{nlmax}(n, m)=2^{n-1}-2^{m+1}$ for $m>$ $\frac{t}{t+k} n-c^{\prime}$ where $c^{\prime}$ is a some constant. Therefore we are interesting to find a proper $(k, k, p, t)$-matrix where the ratio $\frac{t}{k}$ is as small as possible. 
For given positive integer $k$ we denote by $t(k)$ the minimal positive integer $t$ such that for some $p$ there exists a proper $(k, k, p, t)$-matrix. It is clear that we can consider only matrices without all-* columns. Then obviously $p \leq t \cdot 2^{k}$. There exists a proper $(k, k, k, 2 k)$-matrix (all rows are different and without $*$ ). Thus, to find $t(k)$ it is sufficiently to consider only a finite set of matrices.

Proposition 1. Let $k_{1}$ and $k_{2}$ be positive integers. Then $t\left(k_{1}+k_{2}\right) \leq t\left(k_{1}\right)+$ $t\left(k_{2}\right)$.

Proof. By definition for some $p_{1}$ and $p_{2}$ there exist a proper $\left(k_{1}, k_{1}, p_{1}, t\left(k_{1}\right)\right)$ matrix $B^{\prime}$ and a proper $\left(k_{2}, k_{2}, p_{2}, t\left(k_{2}\right)\right)$-matrix $B^{\prime \prime}$. Compose a $\left(2^{k_{1}+k_{2}} \times\left(p_{1}+\right.\right.$ $\left.p_{2}\right)$ ) matrix $B$ where the rows of $B$ are all possible concatenations of rows of matrices $B^{\prime}$ and $B^{\prime \prime}$. It is easy to see that $B$ is a proper $\left(k_{1}+k_{2}, k_{1}+k_{2}, p_{1}+\right.$ $\left.p_{2}, t\left(k_{1}\right)+t\left(k_{2}\right)\right)$-matrix. Therefore $t\left(k_{1}+k_{2}\right) \leq t\left(k_{1}\right)+t\left(k_{2}\right)$.

It is quite obvious that

Proposition 2. $t(k) \geq k$.

Propositions 1 and 2 follow that there exists the limit $\lim _{k \rightarrow \infty} \frac{t(k)}{k}$.

A proper $\left(k_{0}, k, p, t\right)$-matrix $B$ can be interpreted as a collection of $2^{k}$ disjoint subcubes in Boolean cube $\{1,2\}^{p}$. Indeed, a row of $B$ can be interpreted as a subcube where the components with $*$ are free whereas the components with 1 or 2 are substituted by correspondent constant. We illustrate this at the example of the matrix $B_{3,3,4,5}$ :

$\begin{array}{cc}\text { row of } B_{3345} & \text { points of a subcube } \\ * 122 & \{(1,1,2,2),(2,1,2,2)\} \\ 2 * 12 & \{(2,1,1,2),(2,2,1,2)\} \\ 22 * 1 & \{(2,2,1,1),(2,2,2,1)\} \\ 122 * & \{(1,2,2,1),(1,2,2,2)\} \\ 2111 & \{(2,1,1,1)\} \\ 1211 & \{(1,2,1,1)\} \\ 1121 & \{(1,1,2,1)\} \\ 1112 & \{(1,1,1,2)\}\end{array}$

The property (i) of a proper matrix provides that subcubes are disjoint. The properties (ii) and (iii) characterize the location of subcubes in a cube and the size of subcubes.

Estimating the numbers of points at different levels of Boolean cube that belong to some disjoint subcubes we are able to prove that

Proposition 3. $t(1)=2, t(2)=4, t(3)=5, t(4)=6, t(5)=8, t(6)=9$, $t(7)=11, t(8)=12, t(10)=15$.

Already after FSE 2001 Fedorova and Tarannikov analysing a preliminary version of this paper [21] proved in [5] and [6] that there do not exist proper $\left(k_{0}, k, p, t\right)$-matrices for $\frac{t}{t+k}<\frac{1}{\log _{2}(\sqrt{5}+1)}=0.5902 \ldots$ It follows that by means 
of only the method (2) with using of proper matrices it is impossible to construct $m$-resilient function on $V^{n}$ with maximal possible nonlinearity $2^{n-1}-2^{m+1}$ for $m<0.5902 \ldots n+O(1)$. At the same time in [5] and [6] it is proved that $\lim _{k \rightarrow \infty} \frac{t(k)}{k}=\frac{1}{\log _{2}(\sqrt{5}+1)}=0.5902 \ldots$ and it is constructed at infinite sequence of such functions with $m=0.5902 \ldots n+O\left(\log _{2} n\right)$. Nevertheless the smallest parameters given in [5] and [6] that improve our bounds are $n=172, m=102$.

The author is grateful to Claude Carlet, Oktay Kasim-Zadeh and Maria Fedorova for helpful discussions.

\section{References}

1. P. Camion, C. Carlet, P. Charpin, N. Sendrier, On correlation-immune functions, Advances in Cryptology: Crypto '91, Proceedings, Lecture Notes in Computer Science, V. 576, 1991, pp. 86-100.

2. Seongtaek Chee, Sangjin Lee, Daiki Lee and Soo Hak Sung, On the Correlation Immune Functions and their Nonlinearity, Advances in Cryptology - Asiacrypt '96, Lecture Notes in Computer Science, V. 1163, 1996, pp. 232-243.

3. B. Chor, O. Goldreich, J. Hastad, J. Friedman, S. Rudich, R. Smolensky, The bit extraction problem or $t$-resilient functions, IEEE Symposium on Foundations of Computer Science, V. 26, 1985, pp. 396-407.

4. T. W. Cusick, On constructing balanced correlation immune functions, in Sequences and Their Applications, Proceedings of SETA '98, Springer Discrete Mathematics and Theoretical Computer Science, 1999, pp. 184-190.

5. M. Fedorova, Yu. Tarannikov. On the constructing of highly nonlinear resilient Boolean functions by means of special matrices, Cryptology ePrint archive (http://eprint.iacr.org/), Report 2001/083, October 2001, 16 pp.

6. M. Fedorova, Yu. Tarannikov. On the constructing of highly nonlinear resilient Boolean functions by means of special matrices, Progress in Cryptology - Indocrypt 2001, Chennai, India, December 16-20, 2001, Proceedings, Lecture Notes in Computer Science, V. 2247, pp. 254-266, Springer-Verlag, 2001.

7. E. Filiol, C. Fontaine, Highly Nonlinear Balanced Boolean Functions with a Good Correlation Immunity, Advanced in Cryptology, Eurocrypt '98, Helsinki, Finland, Lecture Notes in Computer Sciences, Vol. 1403, 1998, pp. 475-488.

8. S. Maitra, P. Sarkar, Highly nonlinear resilient functions optimizing Siegenthaler's Inequality, Crypto '99, Lecture Notes in Computer Science, Vol. 1666, 1999, pp. 198-215.

9. P. Sarkar, S. Maitra, Construction of nonlinear Boolean functions with important cryptographic properties, In Advanced in Cryptology: Eurocrypt 2000, Lecture Notes in Computer Science, V. 1807, 2000, pp. 485-506.

10. P. Sarkar, S. Maitra, Nonlinearity bounds and constructions of resilient Boolean functions, In Advanced in Cryptology: Crypto 2000, Proceedings, Lecture Notes in Computer Science, V. 1880, 2000, pp. 515-532.

11. E. Pasalic, S. Maitra, T. Johansson, P. Sarkar, New constructions of resilient and correlation immune Boolean functions achieving upper bounds on nonlinearity, WCC2001 International Workshop on Coding and Cryptography, Paris, January 8-12, 2001, Electronic Notes in Discrete Mathematics, Volume 6, Elsevier Science, 2001. 
12. E. Pasalic, T. Johansson, Further results on the relation between nonlinearity and resiliency for Boolean functions, IMA Conference on Cryptography and Coding, Lecture Notes in Computer Science, Vol. 1746, 1999, pp. 35-44.

13. N. J. Patterson, D. H. Wiedemann, The covering radius of the $\left[2^{15}, 16\right]$ ReedMuller code is at least 16276, IEEE Transactions on Information Theory, V. 29, No. 3, pp. 354-356, May 1983.

14. N. J. Patterson, D. H. Wiedemann, Correction to [13], IEEE Transactions on Information Theory, V. 36, No. 2, p. 443, March 1990.

15. O. S. Rothaus, On bent functions, Journal of Combinatorial Theory, Series A20, pp. 300-305.

16. J. Seberry, X. Zhang, Y. Zheng, On Constructions and Nonlinearity of Correlation Immune Functions, Advances in Cryptology, Eurocrypt '93, Proceedings, Lecture Notes in Computer Science, V. 765, 1993, pp. 181-199.

17. T. Siegenthaler, Correlation-immunity of nonlinear combining functions for cryptographic applications, IEEE Transactions on Information theory, V. IT-30, No 5, 1984, p. $776-780$.

18. T. Siegenthaler, Decrypting a Class of Stream Ciphers Using Ciphertext Only, IEEE Transactions on Computer, V. C-34, No 1, Jan. 1985, pp. 81-85.

19. Yu. Tarannikov. On resilient Boolean functions with maximal possible nonlinearity, Cryptology ePrint archive (http://eprint.iacr.org/), Report 2000/005, March 2000, $18 \mathrm{pp}$.

20. Yu. Tarannikov. On resilient Boolean functions with maximal possible nonlinearity, Proceedings of Indocrypt 2000, Lecture Notes in Computer Science, V. 1977, pp. 19-30, Springer-Verlag, 2000.

21. Yu. Tarannikov. New constructions of resilient Boolean functions with maximal nonlinearity, 8th Fast Software Encryption Workshop, Preproceedings, Yokohama, Japan, April 2-4, 2001, pp. 70-81.

22. Yu. Tarannikov, P. Korolev, A. Botev. Autocorrelation coefficients and correlation immunity of Boolean functions, Proceedings of Asiacrypt 2001, Gold Coast, Australia, December 9-13, 2001, Lecture Notes in Computer Science, V. 2248, pp. 460-479, Springer-Verlag, 2001.

23. Y. Zheng, X. M. Zhang, Improved upper bound on the nonlinearity of high order correlation immune functions, Selected Areas in Cryptography, 7th Annual International Workshop, SAC2000, Lecture Notes in Computer Science, V. 2012, pp. 264-274, Springer-Verlag, 2001. 